\title{
Accuracy of the preoperative diagnostic workup in patients with head and neck cancers undergoing neck dissection in terms of nodal metastases
}

\author{
Angéla Horváth ${ }^{1} \cdot$ Péter Prekopp ${ }^{1} \cdot$ Gábor Polony $^{1} \cdot$ Eszter Székely $^{2} \cdot$ László Tamás $^{1} \cdot$ Kornél Dános $^{1}$ (1)
}

Received: 16 July 2020 / Accepted: 24 August 2020 / Published online: 29 August 2020

(c) The Author(s) 2020

\begin{abstract}
Purpose The presence of cervical lymph node metastases is one of the most influential prognostic factors in head and neck squamous cell carcinomas. The management of clinically N0 neck in patients with head and neck cancer remains controversial: elective neck dissection has relatively high morbidity, adversely affecting the quality of life, however, abandoning elective neck dissection is known to compromise overall survival in numerous primaries. The purpose of this study was to evaluate the accuracy of the conventional imaging modalities (CT, MRI, US) and fine-needle aspiration cytology (FNAC) in the detection of lymph node metastases in the neck.

Methods Sixty two patients were included in the study, who underwent primary tumor resection and neck dissection. Preoperative nodal status was compared with postoperative histopathology nodal status. In our retrospective study, we reviewed the patient documentation. Statistical analysis of the data—with descriptive statistics and correlation analysis—was performed with Chi-square test.

Results The sensitivity of conventional imaging modalities and FNAC were $82.8 \%$ and $81.8 \%$, respectively, while specificity were $73.9 \%$ and $100 \%$, respectively. Positive predictive value calculated for imaging modalities and FNAC were $82.8 \%$, $100 \%$, respectively, while negative predictive values were $73.9 \%$ and $66.6 \%$, respectively.

Conclusion Neither the sensitivity of imaging modalities (CT, MRI, US) nor FNAC reached $100 \%$, none of these methods can definitively exclude the presence of regional tumor metastasis. According to these data, no permissive alteration should be allowed from the current guidelines (e.g. NCCN) based on imaging/FNAC examinations regarding the need for elective neck dissection.
\end{abstract}

Keywords Imaging $\cdot$ Head and neck $\cdot$ Squamous cell carcinoma $\cdot$ Ultrasound $\cdot$ FNAC $\cdot$ Neck dissection

\section{Purpose}

The presence of cervical lymph node metastases is one of the most influential prognostic factors in head and neck squamous cell carcinomas. Adequate treatment based on precise preoperative diagnostic work-up is crucial for achieving the desired oncologic outcome [1]. The indication for neck

Kornél Dános

danos.kornel@med.semmelweis-univ.hu

1 Department of Otorhinolaryngology, Head and Neck Surgery, Semmelweis University, Szigony Str. 36, Budapest 1083, Hungary

2 2nd Department of Pathology, Semmelweis University, Budapest, Hungary dissection can be either therapeutic (for clinically $\mathrm{N}+$ necks) or elective (for clinically N0 necks). Most currently available guidelines recommend comprehensive neck dissection (levels I-V.) if preoperative examinations (physical examination, imaging, FNAC) reveal pathologic lymph nodes. Based on classic textbooks, elective treatment of the neck is recommended in clinically negative (cN0) necks when the probability of occult micrometastases exceeds $15-20 \%$, which is embraced by the therapeutic guidelines, easing the everyday clinical decision making for certain tumor sites and stages [2].

Unilateral lymph node dissection is usually recommended, but bilateral dissection is warranted in tumors reaching the midline and due to the bilateral lymphatic drainage in tumors in certain locations (e.g. base of the tongue, soft palate, supraglottic larynx). 
Management of the N0 neck is still a controversial area in the surgical treatment of head and neck tumors. It is known that the number of elective neck dissections in which the postoperative histopathological examinations reveal no metastatic lymph nodes is relatively high, however, the morbidity - such as hypesthesia, lymphedema, decreased head/neck, or arm mobility-of these procedures are also substantial in a varying degree, resulting in a decreased quality of life [3]. Moreover, there are cases when regional recurrence occurs over the years, adversely affecting overall survival. For this reason, careful preoperative investigation, diagnostic algorithms, and therapeutic recommendations are extremely important.

In our study, we aimed to evaluate the reliability of the preoperative investigations of our patients who underwent neck dissections by calculating the specificity, sensitivity, negative-, and positive predictive values regarding the presence/absence of metastatic lymph nodes.

\section{Methods}

All patients enrolled in our study were diagnosed (histologically) and treated between September 2012 and August 2017 at the Department of Otolaryngology and Head-Neck Surgery, Semmelweis University, Hungary. The patients underwent upfront surgical treatment (resection of the primary and neck dissection) for cancers of the oral cavity, oropharynx, hypopharynx, or larynx.

Patients who were not diagnosed (by biopsy) at our tertiary referral center, had primaries in other head and neck sites (e.g. nasopharynx, nasal cavity, paranasal sinuses, salivary gland, or skin, furthermore those unknown primaries), or had previous head and neck oncological treatment were excluded from our study, thus, to maintain sample homogeneity, we had to exclude a large number of patients who underwent salvage surgery or were referred to our department from other hospitals.

The patients underwent head and neck imaging (CT or MRI) to determine the stage of their disease. All imaging studies were performed-according to the Hungarian regulations-at the regional imaging center indicated by the patient's location (home addresses). Then, the patients were referred to the US-guided FNAC clinic of the 2nd Department of Pathology, Semmelweis University to obtain cytologic confirmation of any pathologic lymph node detected by ultrasound-expert cytologists.

In our retrospective study, we evaluated the patients' medical records (discharge summaries, multidisciplinary tumor board (MTB) reports, histological reports, and imaging studies). The results of the preoperative examinations were correlated to the histopathological results of the neck dissection specimens. Both preoperative examination results
(cTNM) and pathological TNM (pTNM) were evaluated according to the UICC 2010 version 7 TNM classification [4]. When reviewing the radiological findings, lymph nodes were considered abnormal if they had previously been considered abnormal by the radiologist if they were larger than $10 \mathrm{~mm}$ in their shortest diameter, contained central necrosis, or had a round shape [5].

Bilateral neck dissections were performed in 12 cases where the preoperative FNAC results were the same for the two sides of the neck. However, in two cases, discordant (one side positive, the other side negative) FNAC results were obtained, while bilateral metastases were reported in the pathological report of the neck dissection specimen. Both cases were considered false negatives in our study.

Both FNAC and imaging were done as part of the clinical routine, so they were performed by various physicians (radiologists and cytologists).

\section{Statistical procedure}

The statistical analysis of the data-with descriptive statistics and correlation analysis-was performed with the Chisquare test (Fisher's exact test) using SPSS Statistics for Mac v.22.0 (IBM Corp., Armonk, NY).

\section{Results}

We identified 62 patients who met the criteria described above. In terms of their gender distribution, we examined 54 men and 8 women. The mean age of the patients at the time of diagnosis was 61 years (43-77 years). Regarding the localization of the primaries, most patients had hypopharyngeal tumors $(32.3 \%)$, followed (in descending order of frequency) by supraglottic (22.6\%), transglottic (21\%), glottic $(9.7 \%)$ laryngeal cancer, oropharyngeal $(9.7 \%)$, oral (3.2\%), and subglottic laryngeal (1.6\%) tumors, respectively (Table 1).

Regarding preoperative stages, $\mathrm{T} 1 \mathrm{~T} 1 \mathrm{a}$ and $\mathrm{T} 1 \mathrm{~b}$ for glottic cancers) stage tumors were the least common, only

Table 1 Distribution of tumors by localization

\begin{tabular}{ll}
\hline Localization & $\%$ \\
\hline Oral & 3.2 \\
Oropharynx & 9.7 \\
Hypopharynx & 32.3 \\
Supraglottic & 22.6 \\
Glottic & 9.7 \\
Transglottic & 21 \\
Subglottic & 1.6 \\
\hline
\end{tabular}


Table 2 Types of surgeries performed

\begin{tabular}{lr}
\hline Therapy & \multicolumn{1}{c}{$\%$} \\
\hline Partial laryngectomy with ipsilateral neck dissection & 27.4 \\
Total laryngectomy with ipsilateral neck dissection & 38.7 \\
Pharyngotomy with ipsilateral neck dissection & 8.1 \\
Transoral resection with ipsilateral neck dissection & 6.5 \\
Partial laryngectomy bilateral neck dissections & 4.8 \\
Total laryngectomy bilateral neck dissections & 14.5 \\
\hline
\end{tabular}

Table 3 Relationship between CT report and pathological lymph node status

\begin{tabular}{lll}
\hline$\%(\mathrm{No})$ & \multicolumn{2}{l}{ Pathology lymph node status (pN) } \\
\cline { 2 - 3 } $\begin{array}{l}\text { Lymph node status described on } \\
\text { the CT }\end{array}$ & Negative & Positive \\
\hline Negative & $72.2(13)$ & $27.8(5)$ \\
Positive & $15.6(5)$ & $84.4(27)$ \\
\hline
\end{tabular}

$4.8 \%$, while $\mathrm{T} 2$, $\mathrm{T} 3$ stage tumors accounted for $32.3 \%$ $-32.3 \%$, slightly less than T4a and T4b stages: $30.6 \%$.

In terms of preoperative $\mathrm{N}$ stage of the tumors, $53.2 \%$ were $\mathrm{N} 0,16.1 \%$ were $\mathrm{N} 1,16.1 \%$ were $\mathrm{N} 2 \mathrm{a}, 8.1 \%$ were $\mathrm{N} 2 \mathrm{~b}, 4.8 \%$ were $\mathrm{N} 2 \mathrm{c}$, and $1.6 \%$ of them were stage N3, so $53.2 \%$ of the patients received elective neck dissection and $46.7 \%$ received therapeutic, comprehensive neck dissection.

The most common therapies recommended by MTB were total laryngectomy with ipsilateral neck dissection (ND) (38.7\%), partial laryngectomy with ipsilateral ND (27.4\%), and total laryngectomy with bilateral NDs (14.5\%). The procedures performed are listed in Table 2.

Concerning the distribution of imaging techniques used for head and neck cancer staging, the majority of patients underwent CT scan (84\%), 10\% had MRI scan, and 3.2\% only had ultrasound scan.

As anticipated, we found a significant $(p<0.001)$ correlation between the nodal stage described by imaging (CT, MRI, US) and the histopathology report (pN1, pN2a, pN2b, $\mathrm{pN} 2 \mathrm{c}, \mathrm{pN} 3$ summarized as $\mathrm{pN}+$ and $\mathrm{N} 0$ as $\mathrm{pN}-$ ). However, the discordance was noteworthy: $73.9 \%$ of the radiologic N0 cases were truly negative, while $26.1 \%$ had false negative preoperative imaging results in terms of the nodal stage (Tables 3, 4, 5, 6).

Although there was a significant association between FNAC results and histopathologic lymph node status $(p<0.001)$, the rate of false-negative cases was surprisingly high: only $66.7 \%$ of neck statuses considered negative by FNAC were proven to be negative by the histopathologic examinations of the surgical specimens. As expected, $100 \%$
Table 4 Relationship between MRI report and pathological lymph node status

\begin{tabular}{lll}
\hline$\%(\mathrm{No})$ & $\begin{array}{l}\text { Pathology lymph node status } \\
(\mathrm{pN})\end{array}$ & Positive \\
\cline { 2 - 3 } $\begin{array}{l}\text { Lymph node status described on } \\
\text { the MRI }\end{array}$ & Negative & $20(1)$ \\
\hline Negative & $80(4)$ & $100(1)$ \\
Positive & 0 & \\
\hline
\end{tabular}

Table 5 Relationship between US report and pathological lymph node status

\begin{tabular}{lll}
\hline$\%(\mathrm{No})$ & $\begin{array}{l}\text { Pathology lymph node status } \\
(\mathrm{pN})\end{array}$ \\
\cline { 2 - 3 } $\begin{array}{l}\text { Lymph node status described on } \\
\text { the US }\end{array}$ & Negative & Positive \\
\hline $\begin{array}{l}\text { Negative } \\
\text { Positive }\end{array}$ & $0(0)$ & $0(0)$ \\
\hline
\end{tabular}

Table 6 Relationship between imaging study report and pathological lymph node status

\begin{tabular}{lll}
\hline$\%(\mathrm{No})$ & \multicolumn{2}{l}{$\begin{array}{l}\text { Pathology lymph node } \\
\text { status }(\mathrm{pN})\end{array}$} \\
\cline { 2 - 3 } $\begin{array}{l}\text { Lymph node status described on the } \\
\text { imaging techniques combined }\end{array}$ & Negative & Positive \\
\hline Negative & $73.9(17)$ & $26.1(6)$ \\
Positive & $17.1(6)$ & $82.9(29)$ \\
\hline
\end{tabular}

Table 7 Relationship between FNAC results and pathological lymph node status

\begin{tabular}{lll}
\hline$\%(\mathrm{No})$ & \multicolumn{2}{l}{$\begin{array}{l}\text { Pathology lymph } \\
\text { node status }(\mathrm{pN})\end{array}$} \\
\cline { 2 - 3 } FNAC & Negative & Positive \\
\hline Negative & $66.7(12)$ & $33.3(6)$ \\
Positive & $0(0)$ & $100(27)$ \\
\hline
\end{tabular}

of the lymph nodes diagnosed positive by the FNAC were also positive histopathologically (Table 7).

Based on the above, the sensitivity of imaging modalities was calculated to be $82.8 \%$, while specificity was $73.9 \%$, the positive predictive value was $82.8 \%$, and the negative predictive value was $73.9 \%$. On the other hand, regarding the FNAC, the sensitivity, specificity, negative and positive (Table 8).

The clinical TNM stage (along with clinical lymph node status - $\mathrm{cN}$ ) is finally established by the multidisciplinary tumor board based on physical examination, imaging, and FNAC results. In our study, $96.6 \%$ of clinically $\mathrm{N}+$ cases 
Table 8 Percentage values for CT separately, imaging techniques combined, and FNAC examinations

\begin{tabular}{llll}
\hline$\%$ & CT* & $\begin{array}{l}\text { Imaging tech- } \\
\text { niques combined }\end{array}$ & FNAC \\
\hline Sensitivity & 84.4 & 82.8 & 81.8 \\
Specificity & 72.2 & 73.9 & 100 \\
Positive predictive value & 84.4 & 82.8 & 100 \\
Negative predictive value & 72.2 & 73.9 & 66.6 \\
\hline
\end{tabular}

*For MRI and US, specificity and sensitivity could not be calculated separately because of low number of participants

were proven to be pathologically positive. However, only $69.7 \%$ of cases preoperatively evaluated as NO had pNO stage based on the histopathological examination of the dissection specimen, accordingly, $30.3 \%$. of cases with cN0 stages were false negatives.

\section{Discussion}

Precise staging workup is crucial before decision making regarding the treatment of head and neck cancer patients. In our work, we aimed to determine the diagnostic accuracy of preoperative imaging modalities (CT, MRI, US) and FNAC in terms of clinical and pathological nodal stages. Previous reports found different diagnostic reliability of certain diagnostic modalities.

Knappe and colleagues compared the US-guided fineneedle aspiration cytology results with the histopathology samples of the patients undergone neck dissections. They found FNAC sensitivity to be $89.2 \%$ and specificity to be $98.1 \%$ [6].

According to Takes et al., the US-guided FNAC had 77\% sensitivity and $100 \%$ specificity in the detection of cervical lymph node metastases. This research highlights that the method has low inter-observer variability-and therefore its widespread use is encouraged [7].

Dammann et al. evaluated the efficiency of CT, MRI, and FDG-PET in the preoperative staging of SCC of the head and neck region. They found CT sensitivity to be $80 \%$, specificity to be $93 \%$, while MRI sensitivity was $93 \%$, specificity was $95 \%$. In the case of ambivalent cases, the study recommended PET for an additional diagnostic procedure [8].

Adams et al. compared the accuracy of conventional imaging studies (US, CT, MRI) with FDG-PET. CT showed $82 \%$ sensitivity and $85 \%$ specificity, while MRI had $80 \%$ sensitivity and $79 \%$ specificity. The ultrasound showed a lower sensitivity with $72 \%$. FDG-PET and conventional imaging showed a statistically significant correlation in the detection of cervical lymph node metastases (PET vs. CT, $p=0.017$; PET vs. MRI, $p=0.012$; PET vs. US, $p=0.0001$ ) [9].
W. van den Brekel et al. performed a study evaluating the value of US and US-guided FNAC for the assessment of N0 neck status. US detected the occult lymph node metastasis with $60 \%$ sensitivity and $77 \%$ specificity, while the US-guided FNAC showed $76 \%$ sensitivity and $100 \%$ specificity. Due to this high accuracy, this work considered US-guided FNAC as an important technique in the N0 neck examination [10].

De Bondt and colleagues performed a meta-analysis comparing US, US-guided FNAC, CT, and MRI in the detection of lymph node metastases in head and neck cancer patients. There was a large variability not only in the accuracy of the techniques but also in the cut-off levels in the size and morphology of the lymph nodes considered as positive. US-guided FNAC showed the highest diagnostic odds ratios ( $\mathrm{DOR}=260)$ compared to $\mathrm{US}(\mathrm{DOR}=40)$, $\mathrm{CT}$ $(\mathrm{DOR}=14)$, MRI $(\mathrm{DOR}=7)$. US showed the highest sensitivity with $87 \%$, while the highest specificity was linked to US-guided FNAC (98\%). The meta-analysis concluded that the US-guided FNAC showed the best diagnostic performance, while the US, CT, and MRI were less effective in this regard [11].

In Sumi et al.'s research, CT and MRI were compared by examining changes in lymph node parenchyma (cancer nest, necrosis, and keratinization). Small-sized (shorter axial diameter less than $10 \mathrm{~mm}$ ) and large-sized (shorter axial diameter $10 \mathrm{~mm}$ or larger) lymph nodes were evaluated separately. In the detection of small metastatic lymph nodes, MRI performed significantly better, sensitivity, specificity, positive predictive value, and negative predictive value were $83 \%, 89 \%, 89 \%$, and $84 \%$, respectively, while those for CT-scan were $68 \%$, $79 \%, 79 \%$, and $72 \%$, respectively. There was no significant difference when examining large lymph nodes between the two imaging techniques (MRI: sensitivity $100 \%$, specificity $98 \%$, positive predictive value $99 \%$, negative predictive value $100 \%$, CT: sensitivity $98 \%$, specificity $89 \%$ positive predictive value $95 \%$, negative predictive value $97 \%$ ) [12].

In Akoglu et al.'s paper, the diagnostic efficacy of conventional imaging (US, CT, MRI) in the detection of cervical lymph node metastasis was compared. The following results were obtained in their research: CT sensitivity $77.7 \%$, specificity $85.7 \%$, positive predictive value $91.3 \%$, negative predictive value: $66.6 \%$, MRI sensitivity $59.2 \%$, specificity $92.8 \%$, positive predictive value $94.1 \%$, negative predictive value: $54.1 \%$, US sensitivity $81.4 \%$, specificity $64.2 \%$, positive predictive value $81.4 \%$, negative predictive value: $64.2 \%$. US and CT performed better than MRI, however, there was no significant difference between them. They also concluded that because of the low negative predictive value, neither method could be used to accurately detect cervical metastases [13].

In their retrospective research, Yoon et al. investigated how effectively CT, MRI, US and FDG-PET-CT, and a 
Table 9 Sensitivity and specificity of FNAC, CT, MRI expressed in percentage

\begin{tabular}{|c|c|c|c|c|c|c|c|}
\hline \multirow[t]{2}{*}{ Authors } & \multirow[t]{2}{*}{ Type of the study } & \multicolumn{2}{|c|}{ FNAC } & \multicolumn{2}{|l|}{$\mathrm{CT}$} & \multicolumn{2}{|l|}{ MRI } \\
\hline & & Sens & Spec & Sens & Spec & Sens & Spec \\
\hline Knappe et al. & Prospective, single institution & 89.2 & 98.1 & & & & \\
\hline Takes et al. & Prospective, multi-institute & 77 & 100 & & & & \\
\hline Dammann et al. & Prospective, single institution & & & 80 & 93 & 93 & 95 \\
\hline Adams et al. & Prospective, single institution & & & 82 & 85 & 80 & 79 \\
\hline Brekel et al. & Prospective, single institution & 76 & 100 & & & & \\
\hline De Bondt et al. & Meta-analysis & 80 & 98 & 81 & 76 & 81 & 63 \\
\hline Sumi et al. & Retrospective,single institution & & & 68 & 79 & 83 & 89 \\
\hline Akoglu et al. & Prospective, single institution & & & 77.7 & 85.7 & 59.2 & 92.8 \\
\hline Yoon et al. & Retrospective, multi-institute & & & 77 & 99.4 & 77 & 99.4 \\
\hline Our research & Retrospective, single institution & 81.8 & 100 & 82.8 & 73.9 & & \\
\hline
\end{tabular}

combination of these modalities detect cervical lymph node metastasis. As a result, they had a sensitivity of $77 \%$ and a specificity of $99.4 \%$ for CT and MRI, sensitivity of $78.4 \%$, and specificity of $98.5 \%$ for US. In conclusion, the results of the study showed that sensitivities and specificities of CT, MR, US, and PET/CT appeared to be similar in the detection of cervical lymph node metastases. The use of the four techniques combination yielded improved sensitivity compared with the single use of these techniques, but without a statistically significant difference [14].

Interpreting our results, we can see that neither the sensitivity of the imaging methods (CT, MRI, US) nor the sensitivity of fine-needle aspiration reached $100 \%$. Also, the rate of false-negative results was relatively high for both methods: $26.1 \%$ for imaging techniques and $33.3 \%$ for FNAC. Thus neither method can definitively establish the presence of cervical metastasis. This is especially important in $\mathrm{T} 1$, T2 stage tumors because the N0 cervical stage is the most common in these cancers.

Our results do not differ substantially from those previously published, underlining that no current diagnostic method is reliable enough to exclude the presence of metastatic cervical lymph nodes (Table 9).

\section{Conclusion}

We cannot decide not to perform an elective neck dissection based on the 'negative' result of the preoperative staging workup, current therapeutic guidelines (e.g. NCCN) should be followed in therapeutic decision-making.

Funding K.D. was supported by the Hungarian Talent Program, Individual Scholarship Award 2019 (NTP-NFTÖ-19-B-0045). Open access funding provided by Semmelweis University.

\section{Compliance with ethical standards}

Conflict of interest The authors declare that they have no conflict of interest.

Ethical approval All procedures performed in studies involving human participants were in accordance with the ethical standards of the institutional and/or national research committee and with the 1964 Helsinki Declaration and its later amendments or comparable ethical standards. This research was approved by the Semmelweis University's Regional, Institutional Scientific and Research Ethics Committee (SE TUKEB 105/2014).

Informed consent Informed consent was obtained from all individual participants included in the study.

Open Access This article is licensed under a Creative Commons Attribution 4.0 International License, which permits use, sharing, adaptation, distribution and reproduction in any medium or format, as long as you give appropriate credit to the original author(s) and the source, provide a link to the Creative Commons licence, and indicate if changes were made. The images or other third party material in this article are included in the article's Creative Commons licence, unless indicated otherwise in a credit line to the material. If material is not included in the article's Creative Commons licence and your intended use is not permitted by statutory regulation or exceeds the permitted use, you will need to obtain permission directly from the copyright holder. To view a copy of this licence, visit http://creativecommons.org/licenses/by/4.0/.

\section{References}

1. De Virgilio A et al (2012) The oncologic radicality of supracricoid partial laryngectomy with cricohyoidopexy in the treatment of advanced N0-N1 laryngeal squamous cell carcinoma. Laryngoscope 122(4):826-833

2. Shah JP (2019) Jatin shah's head and neck surgery and oncology, 5th edn. Elsevier Inc, St. Louis

3. Teymoortash A et al (2010) Postoperative morbidity after different types of selective neck dissection. Laryngoscope 120(5):924-929

4. Sobin LH (2010) TNM classification of malignant tumours, vol 7. Wiley, Hoboken, p 309 (Chichester, West Sussex, UK)

5. Anzai Y, Brunberg JA, Lufkin RB (1997) Imaging of nodal metastases in the head and neck. J Magn Reson Imaging 7(5):774-783 
6. Knappe M, Louw M, Gregor RT (2000) Ultrasonography-guided fine-needle aspiration for the assessment of cervical metastases. Arch Otolaryngol Head Neck Surg 126(9):1091-1096

7. Takes RP et al (1996) Regional metastasis in head and neck squamous cell carcinoma: revised value of US with US-guided FNAB. Radiology 198(3):819-823

8. Dammann F et al (2005) Rational diagnosis of squamous cell carcinoma of the head and neck region: comparative evaluation of CT, MRI, and 18FDG PET. AJR Am J Roentgenol 184(4):1326-1331

9. Adams $\mathrm{S}$ et al (1998) Prospective comparison of 18F-FDG PET with conventional imaging modalities (CT, MRI, US) in lymph node staging of head and neck cancer. Eur J Nucl Med 25(9):1255-1260

10. van den Brekel MW et al (1991) Occult metastatic neck disease: detection with US and US-guided fine-needle aspiration cytology. Radiology 180(2):457-461

11. de Bondt RB et al (2007) Detection of lymph node metastases in head and neck cancer: a meta-analysis comparing US, USgFNAC, CT and MR imaging. Eur J Radiol 64(2):266-272
12. Sumi M et al (2007) Diagnostic performance of MRI relative to CT for metastatic nodes of head and neck squamous cell carcinomas. J Magn Reson Imaging 26(6):1626-1633

13. Akoglu E et al (2005) Assessment of cervical lymph node metastasis with different imaging methods in patients with head and neck squamous cell carcinoma. J Otolaryngol 34(6):384-394

14. Yoon DY et al (2009) CT, MR, US,18F-FDG PET/CT, and their combined use for the assessment of cervical lymph node metastases in squamous cell carcinoma of the head and neck. Eur Radiol 19(3):634-642

Publisher's Note Springer Nature remains neutral with regard to jurisdictional claims in published maps and institutional affiliations. 